\title{
Asociación entre los factores demográficos y socioeconómicos con el estado nutricional en niños menores de 5 años en poblaciones rurales de Colima, México
}

\author{
Mirella Bernabeu Justes ${ }^{a, *}$, Carmen Alicia Sánchez-Ramírez ${ }^{\mathrm{b}}$

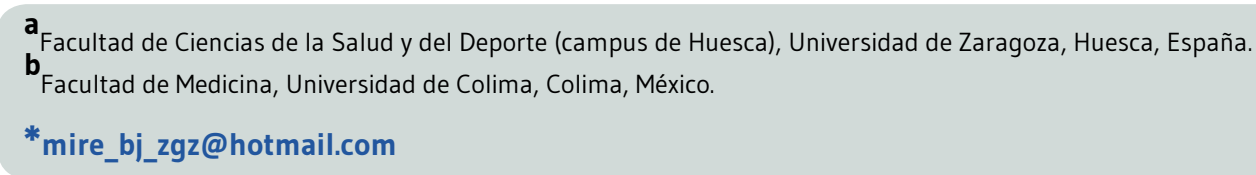

Editora asignada: Carolina Aguirre-Polanco. Pontificia Universidad Católica de Chile. Santiago, Chile.

Recibido el 25 de enero de 2018; aceptado el 18 de junio de 2019; publicado el 30 de junio de 2019.

\section{PALABRAS CLAVE}

Desnutrición;

Sobrepeso;

Obesidad;

Demografía;

Factores

Socioeconómicos.
Asociación entre los factores demográficos y socioeconómicos con el estado nutricional en niños menores de 5 años en poblaciones rurales de Colima, México

\section{RESUMEN}

Introducción: La prevalencia de desnutrición en México se ha mantenido superior en la población rural nacional con respecto a las zonas urbanas. El patrón de crecimiento publicado por la OMS en 2006 confirma que los niños $\leq 5$ años tienen el mismo potencial de crecimiento si las condiciones de vida son óptimas, existiendo diversos factores que pueden afectar el estado nutricional. El objetivo del estudio fue determinar si existe asociación entre los factores demográficos-socioeconómicos con el estado nutricional en poblaciones rurales de Colima.

Material y Métodos: Diseño: transversal analítico. Se incluyeron 72 niños $\leq 5$ años (34 género masculino; 38 femenino) de las poblaciones de Suchitlán, Cofradía de Suchitlán y Zacualpan, (2015 abril-junio). Se realizaron mediciones de peso y talla y se calcularon los indicadores de peso/edad, talla/edad, peso/talla e índice de masa corporal/edad. Se aplicó un cuestionario de factores demográficos y socioeconómicos y se realizó el análisis estadístico usando $\chi^{2} 0$ exacta de Fisher para buscar asociaciones entre el estado nutricional y los factores socioeconómicos o demográficos.

Resultados: La prevalencia de desnutrición crónica fue $25 \%$, desnutrición aguda $2,8 \%$ y sobrepeso/obesidad $11,2 \%$. Se asoció el ser hijo único con el sobrepeso/obesidad $(p=0,016)$ y el recibir lactancia materna con la desnutrición crónica $(\mathrm{p}=0,014)$.

Conclusiones: Se identificó el ser hijo único como factor de riesgo para el desarrollo de sobrepeso/obesidad y la lactancia materna con la presencia de desnutrición crónica. 


\section{KEYWORDS}

Malnutrition;

Overweight;

Obesity;

Demography;

Socioeconomic Factors.

Association between demographic and socioeconomic factors with nutritional status in children under 5 years old in rural populations of Colima, Mexico

\section{AB S T RAC T}

Introduction: The prevalence of malnutrition in Mexico has remained higher in the national rural population than in urban areas. The growth pattern published by the WHO in 2006 confirms that children $\leq 5$ years have the same growth potential if living conditions are optimal, being some factors associated to malnutrition, so the objective of the study was to determine if there is an association between the demographic-socioeconomic factors with nutritional status in rural populations of Colima.

Material and Methods: Design: cross sectional. We included 72 children $\leq 5$ years (34 male gender; 38 female) from the villages of Suchitlán, Cofradía de Suchitlán and Zacualpan (2015, AprilJune). Measurements of weight and height were taken and a questionnaire of demographicsocioeconomic factors was applied to the parents. The indicators weight-for-age, height-forage, weight-for-height and BMl-for-age were calculated. A questionnaire of demographic and socioeconomic factors was applied and the statistical analysis was carried out using $\chi^{2}$ or Fisher's exact to find associations between nutritional status and socioeconomic or demographic factors.

Results: The prevalence of chronic malnutrition was $25 \%$, acute malnutrition $2.8 \%$ and overweight or obesity $11.2 \%$. It was associated being an only child with overweight/obesity $(p=0.016)$ and breastfeeding with chronic malnutrition $(p=0.014)$.

Conclusions: Being an only child was identified as a risk factor for the development of overweight/ obesity and breastfeeding with the presence of chronic malnutrition.

\section{CITA}

Bernabeu Justes M, Sánchez-Ramírez CA. Asociación entre los factores demográficos y socioeconómicos con el estado nutricional en niños menores de 5 años en poblaciones rurales de Colima, México. Rev Esp Nutr Hum Diet. 2019; 23(2): 48-55. doi: 10.14306/renhyd.23.2.545

\section{INTRODUCCIÓn}

El $30 \%$ de la población mundial presenta algún tipo de malnutrición y el $55 \%$ de las muertes que ocurren cada año en los niños están relacionadas con malnutrición. Las complicaciones de la malnutrición son diversas, como infecciones, crecimiento retardado, ceguera, problemas en el desarrollo mental y anemia, entre otras ${ }^{1}$. La forma de desnutrición más frecuente es la desnutrición crónica (baja talla para la edad). Se estima que 178 millones de niños menores de cinco años en el mundo la sufren, la cual es responsable de $35 \%$ de muertes en este grupo de edad ${ }^{2}$. Solamente en América Latina y el Caribe, unos 9 millones de niños y niñas menores de 5 años (el $16 \%$ de los niños de esta edad) sufren de desnutrición crónica y se estima que al menos otros 9 millones de niños están en riesgo de desnutrirse ${ }^{3}$.

México es un país con grandes desequilibrios sociales y en 2010 registró un total de 28.865 muertes en niños menores de 5 años, muchas de las cuales podrían haberse evitado a través del acceso efectivo a instituciones de salud o con acciones sobre el medio ambiente y los determinantes sociales ${ }^{4}$.

Datos publicados por la Encuesta Nacional de Salud y Nutrición (ENSANUT) 2012 revelan que en México la desnutrición infantil ha disminuido. En menores de cinco años la prevalencia de bajo peso para la edad es de $2,8 \%$, la de baja talla para la edad (desnutrición crónica) es de 13,6\% y la de 
emaciación (desnutrición aguda) es de 1,6\%. La baja talla en preescolares disminuyó 13,3 puntos porcentuales entre 1988 y 2012 , al pasar del $26,9 \%$ al $13,6 \%^{4}$.

Las prevalencias de desnutrición en la población rural nacional se han mantenido históricamente al doble que en las zonas urbanas. Respecto a las poblaciones rurales indígenas, entre 1988 y 2012 hubo una disminución de la prevalencia de desnutrición crónica en niños $\leq 5$ años, ya que en 1988 la prevalencia fue de $43,1 \%$ y en el 2012 de 20,9\% (disminuyendo 22,2 puntos porcentuales). En relación a las poblaciones urbanas la prevalencia nacional de desnutrición crónica en 1988 fue de 22,5\% y en el 2012 de 11,1\%, observando que también ha disminuido, pero aún son problemas nutricionales que persisten en México ${ }^{4}$. El período en el que se observó una mayor reducción de la desnutrición fue entre el 1999 y el 2006, que se cree que coincide con el apoyo de programas pertinentes y focalizados por parte del gobierno mexicano a poblaciones vulnerables. A pesar de ello, las cifras siguen permaneciendo más altas en poblaciones rurales, sobre todo en los niños menores de un año de vida.

Aunque el mayor problema de nutrición infantil en las zonas rurales sigue siendo la desnutrición, las prevalencias de sobrepeso y obesidad infantil a nivel mundial han aumentado un 5\% aproximadamente en 1990 y hasta un $7 \%$ en $2012^{5}$. Datos apuntan a que en 2012 había 44 millones de niños menores de 5 años con sobrepeso u obesidad (el 6,7\% del total mundial). En México, la prevalencia de sobrepeso y obesidad en $\leq 5$ años ha aumentado a 9,7\% $\%^{4}$, revelando la transición epidemiológica que está sufriendo el país.

Los niños con sobrepeso no sólo tienen mayor riesgo de convertirse en adultos con sobrepeso, sino que se asocia a factores de riesgo cardiovascular como cifras altas de presión arterial y dislipidemias, entre otros.

El último patrón de crecimiento publicado por la Organización Mundial de la Salud (OMS), en el 2006, confirma que todos los lactantes y niños hasta los 5 años tienen el mismo potencial de crecimiento si las condiciones de vida son similares $^{6}$, y diversos estudios han relacionado diferentes factores demográficos y socioeconómicos con problemas nutricionales, mostrando asociaciones directas con variables como ingresos económicos, escolaridad materna, tipo de familia, entre otros ${ }^{7-20}$. A pesar de las evidencias encontradas, se cree que pudieran diferir los factores en la mayor prevalencia de malnutrición infantil en poblaciones rurales frente a las urbanas, lo cual permitirá llevar a cabo medidas y programas que reduzcan estos desequilibrios y sus consecuencias en el crecimiento y desarrollo infantil, por lo que el objetivo del estudio fue determinar si existe asociación entre los factores demográficos y socioeconómi- cos con el estado nutricional en niños $\leq 5$ años en poblaciones rurales de Colima.

\section{$\longrightarrow$ \\ MATERIAL Y MÉTODOS}

\section{Diseño del estudio y muestra}

El diseño del estudio fue transversal analítico de casos consecutivos. Se incluyeron 72 niños (34 del género masculino y 38 del género femenino) de un mes de edad a 5 años de edad de las poblaciones de Suchitlán (35 niños), Cofradía de Suchitlán (19 niños) y Zacualpan (18 niños) del municipio de Cómala, del Estado de Colima en México, durante abril a junio del 2015. Los criterios de inclusión fueron niños $<5$ años de edad, ambos géneros, sanos y sin antecedentes neonatales de importancia. Se excluyeron aquellos niños que tuvieran diagnóstico de neoplasia, problemas neuroendocrinos, enfermedades crónicas, intolerancias alimentarias o con mediciones incompletas. Los niños se clasificaron en lactantes menores ( $<12$ meses de edad), lactantes mayores ( $12-23$ meses) y preescolares ( $\geq 24$ meses). La variable dependiente fue el estado nutricional y las variables independientes fueron los factores demográficos y socioeconómicos, cuya información se obtuvo a través de un cuestionario realizado a la madre, el padre o el tutor principal.

\section{Evaluación antropométrica}

Se realizó una estandarización antropométrica previa y se tomaron las mediciones de peso en todos los niños; en los niños menores de 2 años se obtuvo la longitud y en los mayores de 2 años la estatura. Los instrumentos que se utilizaron fueron una balanza electrónica portátil (modelo Etekcity 4074C) para el peso, un estadiómetro portátil (modelo Seca 213) para la talla y un infantómetro (modelo Seca 201) para la longitud. La longitud de midió en una superficie plana y con la ayuda de un familiar del niño para mantener la postura del bebé en decúbito supino. Todas las mediciones se realizaron de la misma manera en todas las comunidades.

\section{Estado nutricional}

Para la evaluación del estado nutricional se calcularon los indicadores de peso para la edad, de talla o longitud para la edad, de peso para la talla y de IMC para la edad. El patrón de referencia que se utilizó fue el patrón de la OMS 2007 y los indicadores se interpretaron como puntaje Z. Se consideró normal el puntaje $Z$ de +2 a -2 DE (desviación estándar) y se consideró como malnutrición los valores fuera de dicho rango y malnutrición severa $\leq-3 \mathrm{DE}$. 
El diagnóstico de desnutrición crónica se realizó de acuerdo con la clasificación de la OMS con un puntaje $Z \leq-2$ DE en el indicador talla o longitud para la edad. El diagnóstico de desnutrición aguda se realizó cuando el indicador peso para la talla fue $\leq-2 \mathrm{DE}$. Se realizó el cálculo de índice de masa corporal (IMC) mediante la fórmula peso $(\mathrm{kg}) /[\text { talla }(\mathrm{m})]^{2}$, el diagnóstico de sobrepeso se consideró con valores de IMC para la edad $\geq+2 D E-+3$ DE y de obesidad en valores de $\geq+3$ $D E$. Se consideró como emaciación el valor $\leq-2 \mathrm{DE}$.

\section{Factores demográficos y socioeconómicos}

Las variables independientes fueron los factores demográficos y socioeconómicos, siendo registrados mediante un cuestionario. Dentro de los factores demográficos se estudió el género (femenino/masculino), la edad en meses ( $<12$ meses, $12-23$ meses, $>24$ meses), el grupo etario (lactante, preescolar) y el tipo de familia determinado por el número de hermanos $(0,1-20>$ de 3 hermanos) y el número de progenitores (papá y mamá o sólo un progenitor). En los factores socioeconómicos se estudió la escolaridad del progenitor principal (grado de escolaridad máximo), los ingresos mensuales (suficientes o insuficientes) tomando en cuenta el ingreso de hogar per cápita día del Consejo Nacional de Evaluación de la Política de Desarrollo Social (CONEVAL) del anexo estadístico del 2014 que considera el ingreso corriente total per cápita en los Estados Unidos Mexicanos de 3.460 pesos al mes ${ }^{21} \mathrm{y}$, por último, los antecedentes dietéticos determinados por el antecedente de haber o estar recibiendo lactancia materna y el tiempo que llevaba recibiendo o que recibió lactancia materna.

\section{Análisis estadístico}

Los datos fueron analizados con el programa SPSS. La estadística descriptiva utilizada para las variables cualitativas fue frecuencias y porcentajes, y para variables cuantitativas promedios y desviación estándar. Todas las variables se clasificaron como cualitativas ordinales o nominales y se buscaron asociaciones entre el estado nutricional (normal, desnutrición, sobrepeso/obesidad) con los factores sociodemográficos (ingresos suficientes o insuficientes, hijo único, 2-3 hijos, más de 4 hijos) y dietéticos como (si/no recibió lactancia materna exclusiva los primeros 6 meses) mediante la prueba $\chi^{2}$ o exacta de Fisher en caso necesario. Se consideró como un $p$ significativo aquel $<0,05$.

\section{Consideraciones éticas}

El estudio fue aprobado por el comité de ética de la Facultad de Medicina de la Universidad de Colima, México. Por tratarse de un estudio en menores de edad, se solicitó el consentimiento informado por escrito por parte de los padres o tutores.

\section{RESULTADOS}

El promedio de edad de los niños que ingresaron al estudio fue de $31,5 \pm 17$ meses de edad. Las características sociodemográficas se describen en la Tabla 1 , donde se observa que la mayoría de los niños son preescolares (62,5\%). El 68,1\% tenían uno o dos hermanos y pertenecían a una familia biparental $(83,3 \%)$. La mayoría de los padres

Tabla 1. Factores demográficos y socioeconómicos $(n=72)$.

\begin{tabular}{|c|c|}
\hline Factores & n (\%) \\
\hline $\begin{array}{l}\text { Género } \\
\text { Masculino } \\
\text { Femenino }\end{array}$ & $\begin{array}{l}34(47,2 \%) \\
38(52,8 \%)\end{array}$ \\
\hline $\begin{array}{l}\text { Grupo etario } \\
\text { Lactante menor ( }<12 \text { meses) } \\
\text { Lactante mayor ( } 12-23 \text { meses) } \\
\text { Preescolar ( }>24 \text { meses) }\end{array}$ & $\begin{array}{c}8(11,1 \%) \\
19(26,4 \%) \\
45(62,5 \%)\end{array}$ \\
\hline $\begin{array}{l}\mathbf{N}^{\circ} \text { de hermanos } \\
\text { Hijo único } \\
1-2 \\
3-5\end{array}$ & $\begin{array}{c}15(20,8 \%) \\
49(68,1 \%) \\
8(11,1 \%)\end{array}$ \\
\hline $\begin{array}{l}\mathbf{N}^{\circ} \text { de progenitores } \\
\text { Monoparental } \\
\text { Biparental }\end{array}$ & $\begin{array}{l}12(16,7 \%) \\
60(83,3 \%)\end{array}$ \\
\hline $\begin{array}{l}\text { Escolaridad progenitor principal } \\
\text { Sin estudios } \\
\text { Primaria } \\
\text { Secundaria } \\
\text { Preparatoria } \\
\text { Licenciatura }\end{array}$ & $\begin{array}{c}2(2,8 \%) \\
12(16,7 \%) \\
41(56,9 \%) \\
13(18,1 \%) \\
4(5,6 \%)\end{array}$ \\
\hline $\begin{array}{l}\text { Ingresos } \\
\text { Suficientes } \\
\text { Insuficientes }\end{array}$ & $\begin{array}{l}48(66,7 \%) \\
24(33,3 \%)\end{array}$ \\
\hline $\begin{array}{l}\text { Lactancia materna } \\
\text { Sí } \\
\text { No }\end{array}$ & $\begin{array}{c}67(93,1 \%) \\
5(6,9 \%)\end{array}$ \\
\hline $\begin{array}{l}\text { Tiempo de lactancia materna } \\
<6 \text { meses } \\
6 \text { meses - } 1 \text { año } \\
>1 \text { año }\end{array}$ & $\begin{array}{l}14(20,9 \%) \\
21(31,3 \%) \\
31(47,8 \%)\end{array}$ \\
\hline
\end{tabular}


tenía como máximo nivel de estudios la Secundaria. El ingreso mensual promedio fue de $\$ 4.048,6 \pm 2.045,3$ pesos mexicanos y de acuerdo a la clasificación del CONEVAL para los ingresos mensuales, las familias que percibían ingresos suficientes fueron el doble de las que no. La mayoría de los niños recibieron o estaban recibiendo lactancia materna (93,1\%). El 79,1\% recibieron lactancia materna por lo menos hasta los 6 meses de edad, de los cuales, un 47,8\% continuó recibiéndola después del año de edad.

En la Tabla 2 se describen los valores promedio y DE de cada una de las mediciones antropométricas de los sujetos estudiados por grupo etario. En la Figura 1 se observa el diagnóstico nutricional de la muestra total estudiada $(n=72)$ de acuerdo con los indicadores utilizados, observando que el $25 \%$ de los niños estudiados presentaban desnutrición crónica y un $11,2 \%$ exceso de peso (sobrepeso u obesidad), mientras que en la Tabla 3 se describen las frecuencias de los distintos diagnósticos nutricionales de acuerdo con los grupos etarios, identificando que los preescolares con mayor frecuencia presentaron desnutrición crónica $(31,1 \%)$ y los lactantes mayores sobrepeso + obesidad (21,1\%).

Al realizar el análisis de asociación entre las variables estudiadas se encontró que en los niños que eran hijos únicos la frecuencia de sobrepeso $u$ obesidad $(p=0,016)$ fue mayor que en aquellos niños que tenían hermanos; así mismo, se encontró que aquellos niños que recibieron lactancia materna los primeros 6 meses de edad presentaban con mayor frecuencia desnutrición crónica con respecto a los que recibieron lactancia materna por menos tiempo $(p=0,014)$.

Tabla 2. Mediciones (peso, talla) e índice de masa corporal (IMC) de acuerdo con el grupo etario (lactantes y preescolar).

\begin{tabular}{|c|c|c|c|c|}
\hline \multirow{2}{*}{ Datos antropométricos } & \multicolumn{2}{|c|}{ Por sexo } & \multicolumn{2}{|c|}{ Por grupo etario } \\
\hline & Femenino $(n=38)$ & Masculino $(n=34)$ & Lactantes $(n=27)$ & Preescolares $(n=45)$ \\
\hline $\begin{array}{l}\text { Peso (kg) } \\
\text { Talla (cm) } \\
\text { IMC }\end{array}$ & $\begin{array}{c}13,3 \pm 3,8 \\
89,2 \pm 11,5 \\
16,4 \pm 1,8\end{array}$ & $\begin{array}{c}12,3 \pm 4,7 \\
83,5 \pm 16,1 \\
17,0 \pm 2,3\end{array}$ & $\begin{array}{c}9,3 \pm 2,9 \\
73,3 \pm 9,9 \\
16,9 \pm 2,6\end{array}$ & $\begin{array}{c}15 \pm 3,4 \\
94,5 \pm 9,4 \\
16,6 \pm 1,6\end{array}$ \\
\hline
\end{tabular}

Datos representados como medias y desviación estándar.

Figura 1. Diagnóstico nutricional $(n=72)$.

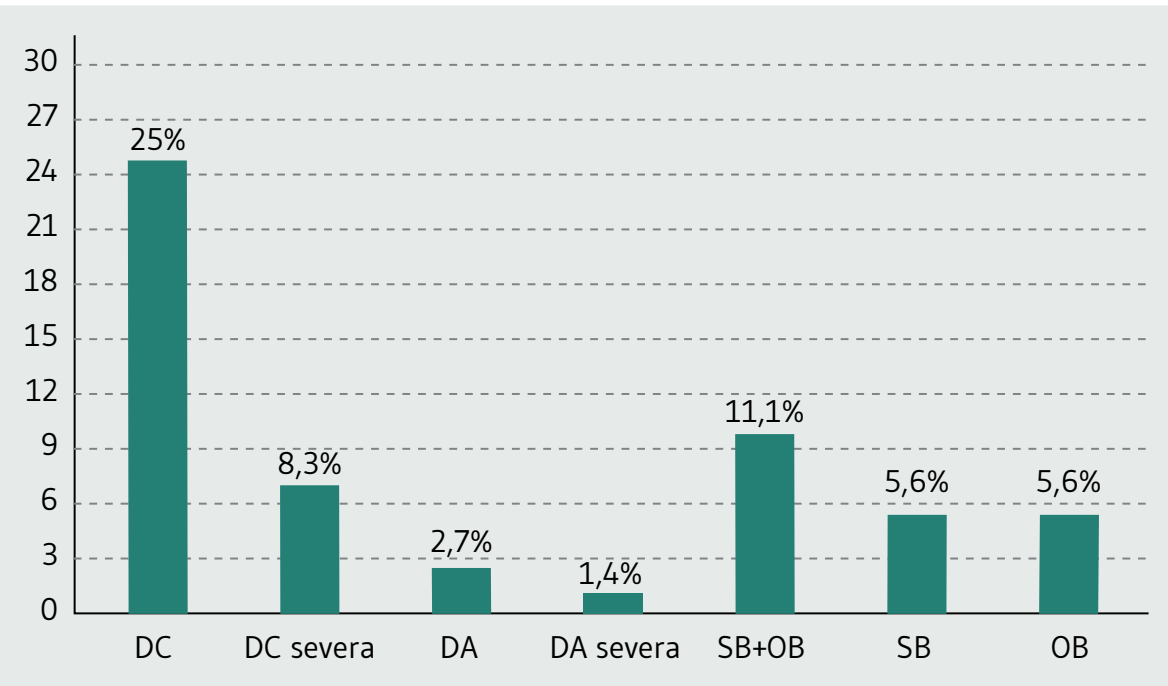

Frecuencia de los distintos tipos de malnutrición de la población total estudiada.

DC: Desnutrición crónica; DA: Desnutrición aguda; SB: Sobrepeso; OB: Obesidad. 
Tabla 3. Diagnóstico nutricional por grupo etario $(n=72)$.

\section{Estado nutricional \\ Desnutrición crónica \\ Lactante menor \\ Lactante mayor \\ Preescolar}

\section{Desnutrición aguda}

Lactante menor

Lactante mayor

Preescolar

\section{Sobrepeso + Obesidad}

Lactante menor

Lactante mayor

Preescolar

Datos representados como frecuencias y porcentajes (\%).

\section{n (\%)}

18 (25\%)

$1(12,5 \%)$

$3(15,8 \%)$

$14(31,1 \%)$

\section{$2(2,8 \%)$}

0

1 (5,3\%)

$1(2,2 \%)$

$8(11,2 \%)$

0

$4(21,1 \%)$

$4(8,9 \%)$

\section{DISCUSIÓN}

Es importante resaltar que la muestra que se incluyó en el presente estudio es pequeña y, por lo tanto, no es representativa de la población estudiada. Sin embargo, se identificaron datos que permiten evaluar la situación nutricional de los menores de 5 años que se incluyeron y viven en las tres poblaciones rurales.

La frecuencia que se identificó de bajo peso y desnutrición crónica en la población estudiada es superior a lo reportado en la ENSANUT 2012. De acuerdo con el Instituto Nacional de Estadística y Geografía (INEGI), las tres comunidades de la muestra pertenecen a la clasificación de pueblos indígenas y considerando los resultados del estado nutricional para la población rural indígena, la frecuencia de desnutrición crónica en el presente estudio está por debajo de lo que se reportó en la ENSANUT, 2012 y en otros estudios ${ }^{14,16}$.

Por grupo etario, la frecuencia de desnutrición crónica en los lactantes menores (menos de 12 meses) en la población estudiada fue 13 puntos porcentuales menos que lo que registra la ENSANUT 2012. La frecuencia de desnutrición crónica en los lactantes mayores (de 12 a 23 meses) de la población estudiada fue similar a los resultados de ENSANUT 2012. En los preescolares (mayores de 24 meses) fue tres veces más a lo que se reporta en la ENSANUT 2012. Así mismo, otros estudios han identificado mayor prevalencia de desnutrición crónica en preescolares ${ }^{7,17}$. Con respecto a la desnutrición aguda o emaciación, se identificó una frecuencia casi del doble, comparado con la ENSANUT 2012.
La frecuencia de sobrepeso/obesidad fue casi dos puntos porcentuales mayor que la que se identificó en la ENSANUT 2012 a nivel nacional, lo que refleja el fenómeno de transición nutricional que está sucediendo en México, dado por el aumento de las cifras de sobrepeso y obesidad y coexistiendo con desnutrición, afectando dicha transición tanto a poblaciones urbanas como rurales ${ }^{18,22}$.

Otros estudios también han mostrado frecuencias mayores de desnutrición crónica en poblaciones rurales respecto a las poblaciones urbanas $9,15,17,19$, similar a lo que nosotros identificamos. Aunque se ha discutido en qué medida la baja talla observada en poblaciones indígenas es producto de la mala nutrición o del somatogenotipo, existen evidencias de que la población indígena con una alimentación adecuada tiende a crecer en forma similar a la norma de referencia ${ }^{15}$.

Respecto a las variables demográficas, hay estudios que también han identificado mayor prevalencia de desnutrición en el sexo femenino ${ }^{8}$, han encontrado asociación entre el número de hermanos y el tipo de familia ${ }^{7,9}$, siendo más frecuente la malnutrición en familias disfuncionales ${ }^{23}$. Así mismo entre la escolaridad de la madre con el estado nutricional $8,9,17,18,20$ y entre los ingresos económicos y la desnutrición $78,18,20$. En el presente estudio se encontró asociación entre la condición obesidad y ser hijo único, al igual que en otros estudios ${ }^{24-26}$, lo que podría estar asociado a la capacidad de consumo de la familia ${ }^{24} \mathrm{y} / \mathrm{o}$ al juego activo en los niños con más de un hermano como medida de prevención del sobrepeso/obesidad ${ }^{26}$.

Con respecto a los antecedentes dietéticos y de acuerdo con las recomendaciones de la OMS, que indica que la lactancia materna exclusiva los primeros seis meses de vida es un factor de protección para el desarrollo de malnutrición infantil7,11-14,16, los resultados de este estudio son contradictorios ya que se encontró que aquellos niños que recibieron lactancia materna al menos los primeros 6 meses de vida presentaron con mayor frecuencia desnutrición crónica. Este resultado debe interpretarse con cautela, ya que el tamaño de muestra de la población estudiada es pequeño y otros factores que no se evaluaron en el presente estudio pudieron haber influido, como la edad a la que se produjo la introducción de alimentación complementaria o los alimentos más frecuentemente consumidos en los primeros dos años de vida. Se ha descrito que los niños que se alimentan exclusivamente con lactancia materna pueden presentar una menor ganancia de peso que los que tienen una alimentación mixta o artificial ${ }^{13}$; además, si estos niños reciben una alimentación complementaria inadecuada a partir de los 6 meses de edad-que no cubra las necesidades energéticas y de nutrientes que la leche materna ya no satisface-, pueden desarrollar malnutrición ${ }^{27}$. Así mismo, hay estudios en los 
que también se ha identificado mayor prevalencia de desnutrición en los niños que habían recibido lactancia materna por más de un año, debido a la incorrecta introducción de la alimentación complementaria ${ }^{10,28}$ o estudios que no encontraron asociación entre el estado nutricional y la lactancia materna ${ }^{29}$.

Es importante resaltar que las limitaciones del estudio son la muestra pequeña, así como el diseño del estudio que sólo permite establecer asociaciones, pero no causalidad. Otra limitación que se debe mencionar es que no se realizó una evaluación de la alimentación complementaria para determinar si esta variable influyó en el estado nutricional de los niños estudiados.

Como perspectiva se considera necesario realizar un estudio similar evaluando otras comunidades rurales que nos permitan continuar haciendo comparaciones y con una muestra mayor que sea representativa.

\section{conclusiones}

Se identificó como factor de riesgo la condición de ser hijo único para el desarrollo de sobrepeso/obesidad y el haber recibido lactancia materna con la presencia de desnutrición crónica.

\section{$\longrightarrow$ \\ CONFLICTO DE INTERESES}

Las autoras expresan que no existen conflictos de interés al redactar el manuscrito.

\section{REFERENCIAS}

(1) Organización de las Naciones Unidas para la Agricultura y la Alimentación. El espectro de la malnutrición. Roma: FAO; 2001. Disponible en: http://www.fao.org/worldfoodsummit/ spanish/fsheets/malnutrition.pdf

(2) Black RE, Allen LH, Bhutta ZA, Caulfield LE, de Onis M, Ezzati $M$, et al. Maternal and child undernutrition: global and regional exposures and health consequences. Lancet. 2008; 371(9608): 243-60.

(3) Programa Mundial de Alimentos. Desnutrición Infantil: Su erradicación es posible. Panamá: WFP; 2009. Disponible en: https://documents.wfp.org/stellent/groups/public/ documents/liaison_offices/wfp205623.pdf
(4) Gutiérrez JP, Rivera-Dommarco J, Shamah-Levy T, VillalpandoHernández S, Franco A, Cuevas-Nasu L, et al. Encuesta Nacional de Salud y Nutrición 2012. Resultados Nacionales. Cuernavaca: Instituto Nacional de Salud Pública (MX), 2012. Disponible en: https://ensanut.insp.mx/informes/ ENSANUT2012ResultadosNacionales.pdf

(5) Organización Mundial de la Salud. Estadísticas Sanitarias Mundiales 2014. Ginebra: OMS; 2014. Disponible en: https://apps.who.int/iris/bitstream/ handle/10665/131953/9789240692695_spa.pdf;jsessionid= 654F80B4A4BB37E2D82F39E70887F590? sequence=1

(6) Organización Mundial de la Salud. Patrones de crecimiento infantil de la OMS: Longitud/estatura para la edad, peso para la edad, peso para la longitud, peso para la estatura e índice de masa corporal para la edad. Métodos y desarrollo. Organización Mundial de la Salud; 2006. Disponible en: https://www.who.int/childgrowth/standards/tr_summary_ spanish_rev.pdf

(7) R A, Sivanandham R, Salome SD, Francis R, D R, Sampavi S, et al. Nutritional Status of Children Aged 3-6 Years in a Rural Area of Tamilnadu. J Clin Diagn Res. 2014; 8(10): JC01-4.

(8) Correia LL, Silva AC e, Campos JS, Andrade FM de O, Machado MMT, Lindsay AC, et al. Prevalence and determinants of child undernutrition and stunting in semiarid region of Brazil. Rev Saude Publica. 2014; 48(1): 19-28.

(9) Arias M, Tarazona MC, Lamus F, Granados C. Estado nutricional y determinantes sociales asociados en niños Arhuacos menores de 5 años de edad. Rev salud pública. 2013; 15: 613-25.

(10) Akombi BJ, Agho KE, Hall J], Merom D, Astell-Burt T, Renzaho AMN. Stunting and severe stunting among children under-5 years in Nigeria: A multilevel analysis. BMC Pediatr. 2017; 17(1): 15.

(11) Aguilar Cordero M], Sánchez López AM, Madrid Baños N, Mur Villar N, Expósito Ruiz M, Hermoso Rodríguez E. Lactancia materna como prevención del sobrepeso y la obesidad en el niño y el adolescente: revisión sistemática. Nutr Hosp. 2015; 31(2): 606-20.

(12) Jarpa MC, Cerda L, Terrazas MC, Cano CC. Lactancia materna como factor protector de sobrepeso y obesidad en preescolares. Rev Chil Pediatr. 2015; 86(1): 32-7.

(13) Morán Rodríguez M, Naveiro Rilo JC, Blanco Fernández E, Cabañeros Arias I, Rodríguez Fernández M, Peral Casado A. Prevalencia y duración de la lactancia materna: Influencia sobre el peso y la morbilidad. Nutr Hosp. 2009; 24(2): 213-7.

(14) Brahm P, Valdés V. Beneficios de la lactancia materna y riesgos de no amamantar. Rev Chil Pediatr. 2017; 88(1): 7-14.

(15) Avila-Curiel A, Shamah-Levy T, Galindo-Gómez C, RodríguezHernández G, Barragán-Heredia LM. La desnutrición infantil en el medio rural mexicano. Salud pública Méx. 1998; 40: 150-60.

(16) Senbanjo IO, Olayiwola IO, Afolabi WAO. Dietary practices and nutritional status of under-five children in rural and urban communities of Lagos State, Nigeria. Niger Med J. 2016; 57(6): 307-13.

(17) Mittal A, Singh J, Ahluwalia SK. Effect of maternal factors on nutritional status of 1-5-year-old children in urban slum population. Indian J Community Med. 2007; 32(4): 264.

(18) Leroy JL, Habicht J-P, González de Cossío T, Ruel MT. Maternal education mitigates the negative effects of higher income on 
the double burden of child stunting and maternal overweight in rural Mexico. J Nutr. 2014; 144(5): 765-70.

(19) Monárrez J, Martínez H. Prevalencia de desnutrición en niños tarahumaras menores de cinco años en el municipio de Guachochi, Chihuahua. Salud pública Méx. 2000; 42: 8-16.

(20) Janevic T, Petrovic O, Bjelic I, Kubera A. Risk factors for childhood malnutrition in Roma settlements in Serbia. BMC Public Health. 2010; 10: 509.

(21) Consejo Nacional de Evaluación de la Política de Desarrollo Social. Informe de pobreza en México 2014. Mexico, DF: CONEVAL; 2016. Disponible en: https://www.coneval.org. $\mathrm{mx} /$ InformesPublicaciones/Documents/Informe-pobrezaMexico-2014.pdf

(22) García-Parra E, Ochoa-Díaz-López H, García-Miranda R, Moreno-Altamirano L, Morales H, Estrada-Lugo EIJ, et al. Estado nutricio de dos generaciones de hermanos(as) < de 5 años de edad beneficiarios(as) de Oportunidades, en comunidades rurales marginadas de Chiapas, México. Nutr Hosp. 2015; 31(6): 2685-91.

(23) González-Rico JL, Vásquez-Garibay EM, Cabrera-Pivaral CE, González-Pérez G], Troyo-Sanromán R. La disfunción familiar como factor de riesgo para obesidad en escolares mexicanos. Rev Med Inst Mex Seguro Soc. 2012; 50(2): 127-34.
(24) Loaiza MS, Atalah SE. Factores de riesgo de obesidad en escolares de primer año básico de Punta Arenas. Rev Chil Pediatr. 2006; 77(1): 20-6.

(25) Girón K, Espinoza L. Epidemiología de la Obesidad en la Consulta Externa de Endocrinología del Departamento de Pediatría. Hospital de Especialidades del Instituto Hondureño de Seguridad Social (IHSS). Período 2002-2004. Rev Med Post Grad Med UNAH. 2006; 9(2): 243-8.

(26) Padilla IS. Prevalencia de sobrepeso-obesidad y factores asociados con valor predictivo-preventivo en escolares de 6 a 11 años de Río Gallegos, Santa Cruz, Argentina. Salud Colectiva. 2011; 7: 377-88.

(27) Asociación Mexicana de Pediatría A. C. Primer Consenso Nacional sobre Alimentación en el Primer Año de la Vida. Acta Pediatr Mex. 2007; 28(5): 213-41.

(28) Tiwari R, Ausman LM, Agho KE. Determinants of stunting and severe stunting among under-fives: evidence from the 2011 Nepal Demographic and Health Survey. BMC Pediatr. 2014; 14: 239.

(29) Campos CC, André MC, Fernández JLM, Grávalos GJD. Prevalencia de obesidad infantil y lactancia materna. Rev Enferm CyL. 2015; 7(1): 80-7. 